\title{
Ein Verfahren zum Nachweise von Kokosfett in Butter.
}

\author{
Von \\ Prof. Dr. H. P. Wijsman und Dr. J. J. Reijst. \\ Mitteilung aus dem Pharmazeutischen Laboratorium der Universitat \\ Leiden.
}

Die Verfahren zum Nachweise des Kokosfettes in Butter benutzen entweder qualitative Merkmale, wie Krystallisationsverhälnnisse, oder quantitative Bestimmungen von gewissen Anteilen der Fettsäuren, hauptsächlich der flüchtigen. Obgleich es an und für sich etwas miBlich ist, auf die An-oder Abwesenheit eines bestimmten Körpers aus dem Gehalt an einem Bestandteil, der nicht für diesen Körper ausschließlich charakteristisch ist, zu schließen, so sind dennoch in diesem Falle die quantitativen Methoden schwer zu umgehen, weil es eben im Kokosfette, soweit wir wissen, einen derartigen charakteristischen Bestandteil, wie er z. B. im Sesamöl oder in Baumwollsamenöl enthalten ist, nicht gibt. Das Kokosfett unterscheidet sich von den übrigen Speisefetten bekanntlich durch den hohen Gehalt an Säuren zwisehen $\mathrm{C}_{6}$ und $\mathrm{C}_{12}$. Wie der eine von uns ${ }^{1}$ ) nachgewiesen hat, fehlen in ihm einerseits die Buttersäure, andererseits die Palmitin- und Stearirsäure. Da bei der Bestimmung der flüchtigen Fettsäuren durch Destillation die Laurinsäure nur sehr unvollständig ausgetrieben wird, liegt es auf der Hand, den Nachwejs des Kokosfettes auf die Bestimmung der Kapron-, Kapryl- und Kaprinsäure zu gründen.

Unsere diesbezüglichen Arbeiten waren schon ziemlich weit gediehen, als die interessante Abhandlung von Orla Jensen ${ }^{2}$ ) erschien, welche zu den wichtigsten zählt, welche seit Jahren über Butteruntersuchung veröffentlicht sind. Obgleich wir seinen Auseinandersetzungen in theoretischer Hinsicht eigentlich nichts hinzuzufügen haben, glauben wir dennoch auf demselben Wege, den auch er gegangen, zu einer Methode gelangt zu sein, die sich den praktischen Verhältnissen der Butteruntersuchung in ihrer üblichen Gestaltung gut anschließt. Wir benutzen die Eigenschaften der Silbersalze, wie auch Kirschner ${ }^{3}$ ) es getan hat. Seine Methode scheint zwar in theoretischer Hinsicht wenig empfehlenswert für den Nachweis von Kokosfett in Butter; sie ist aber auch äberhaupt nicht zu diesem Zweeke von ibm ausgearbeitet. Kirschner unterwirft das neutralisierte Destillat von der Bestimmung der Reichert-Meißl'schen Zahl aufs neue dieser selben Bestimmung, nachdem er die als Silbersalz fällbaren Säuren daraus entfernt hat. Es ist aber genügend bekannt, daß bei dieser Bestimmung nur ein gewisser Teil der flüchtigen Fettsäuren in das Destillat übergebt, dessen Gleichheit durch ein peinliches Innehalten der Ausführungsbedingungen gesichert werden muB. Die Arbeit Jensen's hat erst recht gezeigt, von welchen Umständen die Größe des übergehenden Anteils beeinfluBt wird. Es scheint also einfacher, wenn man untersuchen will, welcher Anteil der flüchtigen Fettsäuren im Reichert-MeiBl'schen Destillate durch Silber fällbar ist, die Fällung mit einer bekannten Menge ${ }^{1 / 10}$ N.-Silberlösung vorzunehmen, und das überschüssige Silber, nach-

1) J. J. Reijst, Het kokosvet, bijdrage tot de kennis der vetten en vetzuren. Dissertation, Leiden 1905.

2) Diese Zeitschrift 1905, 10, 265.

3) Diese Zeitschrift 1905, 9, 65 . 
dem die fettsauren Silbersalze abfiltriert und gewaschen sind, mit $1 / 10 \mathrm{~N}$.-Chlornatrium nach Mohr zu titrieren. Werden alle Zahlen in $\mathrm{ccm} \mathbf{1}_{10} \mathrm{~N}$.-Iösungen angegeben und auf $5 \mathrm{~g}$ Butterfett bezogen, so erreicht man in dieser Art eine Zergliederung der Reichert-Meißl'schen Zahl in zwei Teile, deren einer die sämtlichen durch Silber fällbaren Fettsäuren darstellt. Wir wollen diese Zahl im folgenden die Silberzahl nennen. Sie ist ungefähr das, was $O$. Jensen die Kaprylsäurezahl nennt, aber nicht ganz. Jensen will nämlich eine vollständige Analyse der gefällten Silbersalze ausführen und nur die Kaprylsäure bestimmen. Denn nur dadurch, meint er, läßt sich mehr erreichen als mit den üblichen Schnellmethoden. Wir behaupten dagegen, daß, wenigstens für die praktischen Zwecke der Butteruntersuchung, eine Schnellmethode, welche die gesamte Gruppe der von Silber fällbaren flüchtigen Fettsäuren zu bestimmen gestattet, von Nutzen sein kann. Da sich an dieser Fällung aber bekanntlich nicht die Kaprylsäure allein beteiligt, scheint uns die nichts vorwegnehmende allgemein gehaltene Bezeichnung „Sil berzahl“ angezeigt.

Um eine Vorstellung von der Größe dieser Zahlen zu geben, bringen wir einige Beispiele:

Es wurden z. B. bei Butterproben gefunden:

$\begin{array}{lcccccccc}\text { Reichert-Meifl'sche Zahl } & 31,8 & 31,4 & 30,28 & 29,6 & 29,5 & 27,9 & 27,7 & 19,25 \\ \text { Silberzahl . . . . . . . . } & 5,06 & 5,55 & 5,27 & 5,11 & 4,79 & 5,40 & 4,62 & 2,10\end{array}$

Es fragt sich nun, in welchem Grade die verschiedenen Fettsäuren im ReichertMeißß l'schen Destillate sich an der Silberfällung beteiligen, Aus den Löslichkeitsverhältnissen des Silberbutyrats ergibt sich, dab die Buttersäure in neutralisierten Destillate von der Bestimmung der Reichert-MeiBl'schen Zahl von einer 1/10 N.-Silberlösung nicht gefällt wird, selbst wenn die sämtlichen flüchtigen Fettsäuren einer Butter mit hoher Reichert-Meißl'schen Zahl als Buttersäure in Rechnung gezogen werden und wenn der Einflub eines Überschusses der Silberlösung auf die Dissoziation beachtet wird. Eine Butter z. B. mit der Reichert-Meißl'schen Zahl 33 gibt $130 \mathrm{ccm}$ neutralisiertes Destillat. Wenn $40 \mathrm{~cm} \mathrm{1/10} \mathrm{N.-Silbernitrat} \mathrm{zugefügt} \mathrm{werden,} \mathrm{so} \mathrm{ist} \mathrm{das}$ Gesamtvolumen $170 \mathrm{~cm}$ und wenn sämtliche Säure Buttersäure wäre, würde die Konzentration des Silberbutyrats 3/170-normal sein, während, selbst bei einem Überschuß von Silber-Ionen, nach Jensen die Löslichkeit des Silberbutyrats noch $1 / 56$-normal ist,

Die Löslichkeit der Silbersalze der Kapryl- und Kapronsäure ist dagegen genügend gering, um eine Bestimmung, namentlich der ersteren, zu gestatten. Bei Gegenwart von Silbernitrat im Überschuß ist wach Jensen Silberkapronat nur zum Betrage von 0,0013-normal löslich, Silberkaprylat sogar nur 0,0002 normal. Da eine gesättigte Kaprylsäurelösung 0,0056-normal ist, werden also fast $97 \%$ der Säure durch Silber gefällt, und auch in nicht gesättigter Lösung immer noch die Hauptmenge.

Tatsächlich fanden wir auch in einer Mischung von $25 \mathrm{ccm} 1 / 10$ N.-Buttersäure und $100 \mathrm{ccm}$ gesättitigter Kaprylsäurelösung, durch Titrierung des überschüssigen Silbers in der angegebenen Weise, 97\% der Kaprylsäure im Silberniederschlage zurück.

Gesättigte Kapronsäurelösung ist 0,078-normal; diese Säure wird also aus gesättigten bis halb gesättigten Lösungen auch zu etwa 97 bis $95 \%$ gefällt; wir fanden etwas weniger als die Rechnung ergibt, nämlich $86 \%$. Da ferner die Kaprinsäure fast unlöslich ist, können wir also schliessen, daf an der Silberzahl beinahe nur die Kapron- und Kaprylsäure beteiligt sind, und zwar von diesen fast die ganze vorhandene Menge, oder aber, wenn ihre Konzentration nicht sehr gering ist, jedenfalls mehr wie $90 \%$ 
Für den Nachweis von Kokosfett in Butter läßt sich nun aber die Silberzahl nicht in der Weise verwenden, daß man an größeren Reihen Butterproben ihren Wert im Destillate von der Bestimmung der Reichert-Meibl'schen Zahl bestimmt, and so zu Grenzzahlen zu kommen versucht. Denn bei irgend einem etwas bohen Gehalt an Kaprylsäure reicht die Menge von $110 \mathrm{ccm}$ Destillat nicht aus, um die überdestillierte Säure in Lösung zu halten. Tatsächlich sieht man dann auch bei der Bestimmung der Reichert-MeiBl'schen Zahl in einer Fettmischung, die Kokosfett enthält, auf der Oberfläche des Destillates Tröpfchen schwimmen; diese enthalten die Hauptmenge der Kaprylsäure, welche eben für das Kokosfet charakteristiseh ist, und diese wird dann beim Filtrieren entfernt. Wenn dazu noch die Tröpfehen irgend bedeutende Mengen von Kaprinsäure enthalten, welche an sich fast unlöslich ist, so tritt diese sogar, wie Jensen dargetan hat, den niedrigeren Fettsäuren gegenüber als eine Art Ausschüttelungsflüssigkeit auf, und hält sehr merkliche Mengen dieser Säuren zurück, welche sonst sich im Wasser gelöst haben würden. Jensen hat den bedeutenden Einfluß dieser Verhältnisse auf die Zahlen nachgewiesen, welche man bekommt, wenn die Bestimmung der Reichert-Meibl'schen Zahlen mit abweichenden Fettmengen ausgeführt und nachher auf $5 \mathrm{~g}$ Substanz umgerechnet werden.

Man kann aber bei der Bestimmung der Reichert-MeiBl'schen Zabl die Menge des Destillats vermehren, zum Beispiel auf $300 \mathrm{ccm}$ und so bewirken, daß die Kaprylsäure, um welche es sich hierbei ja gerade handelt, in Lösung bleibt und nicht beim Filtrieren fortgeschafft wird. Die Silburzahl, in diesem größeren Destillate bestimmt, wird dann höher sein, wie die Silberzahl in den $110 \mathrm{ccm}$ Destillat. Bei reinem Butterfett dagegen ist, soweit wir wissen, die Kaprylsäure nicht in so großer Menge vorhanden, daß sie im Destillate von $110 \mathrm{ccm}$ nicht vollständig löslich wäre. Durch Vermehrung des Destillates wird also die Silberzahl auch keine Steigerung erfahren, vielmehr etwas erniedrigt werden, da von dem Silberniederschlage im größeren Volumen Flüssigkeit etwas mehr gelöst bleiben kann.

Wir verfabren also in folgender Weise:

Es wird in gewöhulicher Weise die Reichert-MeiBl'sche Zahl bestimmt, wobei die Glycerin-Natron-Verseifung vorzuziehen ist.

$\mathrm{Zu}$ dem Filtrate werden nach der Titration $40 \mathrm{~cm} 1 / 10 \mathrm{~N}$ N.Silbernitratlösung zugefügt; darauf wird filtriert und der Niederschlag bis auf etwa $200 \mathrm{~cm}$ Filtratmenge ausgewaschen. Zur Flüssigkeit werden $50 \mathrm{ccm} 1 / 10 \mathrm{~N}$.-ChlornatriumIösung und 2 Tropfen einer gesättigten Kaliumehromatlösung hinzugefügt und der Überschuß an Chlornatrium mit 1/10 N.-Silberlösung zurücktitriert. Der Unterschied zwischen den im ganzen verwendeten com $1 / 10 \mathrm{~N}$.-Silberlösung und den ccm 1/10 N.-Chlornatriumlösung, erhöht um $1 / 10$, ist die „Erste Silberzahl“.

Es wird nun eine zweite Reichert-MejBl'sche Zahl bestimmt in der Art, daß, nachdem je etwa $100 \mathrm{ccm}$ Flüssigkeit überdestilliert sind, aufs neue zweimal $100 \mathrm{ccm}$ Wasser durch einen Hahntrichter in den Destillierkolben gegeben werden, und die Destillation fortgesetzt, bis im ganzen $300 \mathrm{ccm}$ Destillat erhalten sind. Das Destillat wird umgeschüttelt, filtriert und darauf werden $250 \mathrm{ccm}$ des Filtrates unter Anwendung von Phenolphthalein als Indikator wie bei der Bestimmung der Reichert-MeiBl'schen Zahl neutralisiert. Zu der neutralisierten Flüssigkeit werden $40 \mathrm{~cm} 1 / 10 \mathrm{~N}$.-Silberlösung zugefügt, der Niederschlag wird abfiltriert, bis auf etwa $350 \mathrm{ccm}$ Filtrat ausgewaschen und im Übrigen wird wie oben verfahren. Die erhaltene $Z$ ahl, erhöht um ${ }_{i}^{1}$, ist die "Zweite SilberzahI". 
Ist die zweite Silberzahl höher wie die erste, so darf auf die Anwesenheit von Kokosfett geschlossen werden.

Es bleibt bei dieser Arbeitsweise die absolute Höhe der Silberzahl außer Betracht; nur das VerhäItnis der beiden Silberzahlen ist maßgebend. Wir sehen dies als einen Vorzug der Methode an; denn die Erfahrung hat genügend dargetan, welchen Unannehmlichkeiten, um es milde auszudrücken, man sich aussetzt, wenn man ein in seinen Eigenschaften so schwankendes Naturprodukt wie die Butter, durch die Aufstellung von Grenzzahlen charakterisieren will, und nachher als abnormal definiert werden mub, was sich den verfrühten Schlüssen des Chemikers nicht fügt. Es scheint also besser, die Aufstellung von Grenzzahlen zu umgehen und nur das Verhält$\mathrm{n}$ is der Ergebnisse der beiden Destillationen in Betracht zu ziehen.

Wir geben zuerst als Beispiel die Werte von zwei Butterproben von unbedingter Reinheit, deren erstere aber durch die abnormalen Zahlen für Refraktion und flüchtige Fettsäuren den Verdacht der Fälschung mit Kokosfett erwecken könnte, während die zweite sich durch eine anormal niedrige Reichert-Meibl'sche Zahl auszeichnet. Es wurde gefunden:

\begin{tabular}{|c|c|}
\hline Refraktion & 42,6 \\
\hline Rejchert-Veilal'sche Zahl & 25,0 \\
\hline Erste Silberzahl & 4,73 \\
\hline Zweite Silberzahl & 4,62 \\
\hline
\end{tabular}

Es zeigt sich, daß diese sogenannten anormalen Butterproben sich unserer Methode gegenüber sehr normal verhielten.

Wir geben nun die Untersuchungsergebnisse bei einer Reihe von Butterproben wieder, denen 5 und $10 \%$ Kokosfett zugemischt wurden.

\begin{tabular}{|c|c|c|c|c|c|}
\hline \multirow{2}{*}{ Nähere Bezeichnung } & & \multicolumn{2}{|c|}{ FJüchlige F'ettsäuren } & \multicolumn{2}{|c|}{ Silberzahl } \\
\hline & & $\begin{array}{l}\text { in }[10 \mathrm{ecm} \\
\text { Destillat } \\
\left(\mathrm{K}_{*}, \mathrm{M}, \sim \mathrm{Zahl}\right)\end{array}$ & $\begin{array}{c}\text { in } 300 \mathrm{ccm} \\
\text { Destillat }\end{array}$ & $\begin{array}{c}\text { in } 110 \mathrm{cem} \\
\text { Destillat }\end{array}$ & $\begin{array}{c}\text { in } 300 \mathrm{ccm} \\
\text { Destillat }\end{array}$ \\
\hline $\begin{array}{l}\text { Butter I.*. } \\
\text { Desgl. mit }\left\{\begin{array}{cc}5 \% & * \\
10 \% & \text { Kokosfett }\end{array}\right.\end{array}$ & $\cdot$ & $\begin{array}{l}21,1 \\
18,7 \\
18,9\end{array}$ & $\begin{array}{l}24,4 \\
25,0 \\
25,1\end{array}$ & $\begin{array}{l}3,1 \\
5,5 \\
5,4\end{array}$ & $\begin{array}{l}2,6 \\
6,2 \\
7,8\end{array}$ \\
\hline 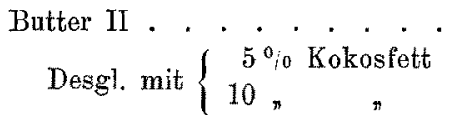 & $\begin{array}{l}\cdot \\
\cdot\end{array}$ & $\begin{array}{l}21,5 \\
19,7 \\
19,9\end{array}$ & $\begin{array}{l}26,9 \\
26,9 \\
27,0\end{array}$ & $\begin{array}{l}4,1 \\
3,6 \\
5,1\end{array}$ & $\begin{array}{l}3,7 \\
7,1 \\
8,3\end{array}$ \\
\hline  & $\begin{array}{l}\cdot \\
.\end{array}$ & $\begin{array}{l}22,55 \\
22,1 \\
21,0\end{array}$ & $\begin{array}{l}25,1 \\
25,6 \\
25,6\end{array}$ & $\begin{array}{l}4,8 \\
4,0 \\
4,8\end{array}$ & $\begin{array}{l}4,45 \\
8,8 \\
9,2\end{array}$ \\
\hline 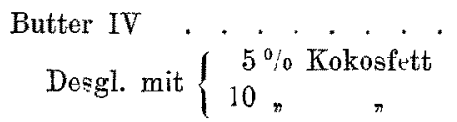 & $\begin{array}{l}. \\
.\end{array}$ & $\begin{array}{l}22,6 \\
22,2 \\
21,7\end{array}$ & $\begin{array}{l}26,2 \\
27,5 \\
27,4\end{array}$ & $\begin{array}{l}4,5 \\
4,1 \\
5,1\end{array}$ & $\begin{array}{l}4,3 \\
8,2 \\
8,4\end{array}$ \\
\hline 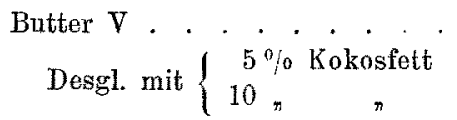 & $\begin{array}{l}\cdot \\
\cdot\end{array}$ & $\begin{array}{l}23,4 \\
28,1 \\
23,0\end{array}$ & $\begin{array}{l}27,0 \\
27,4 \\
28,6\end{array}$ & $\begin{array}{l}4,8 \\
5,8 \\
5,5\end{array}$ & $\begin{array}{l}4,8 \\
7,4 \\
8,3\end{array}$ \\
\hline
\end{tabular}




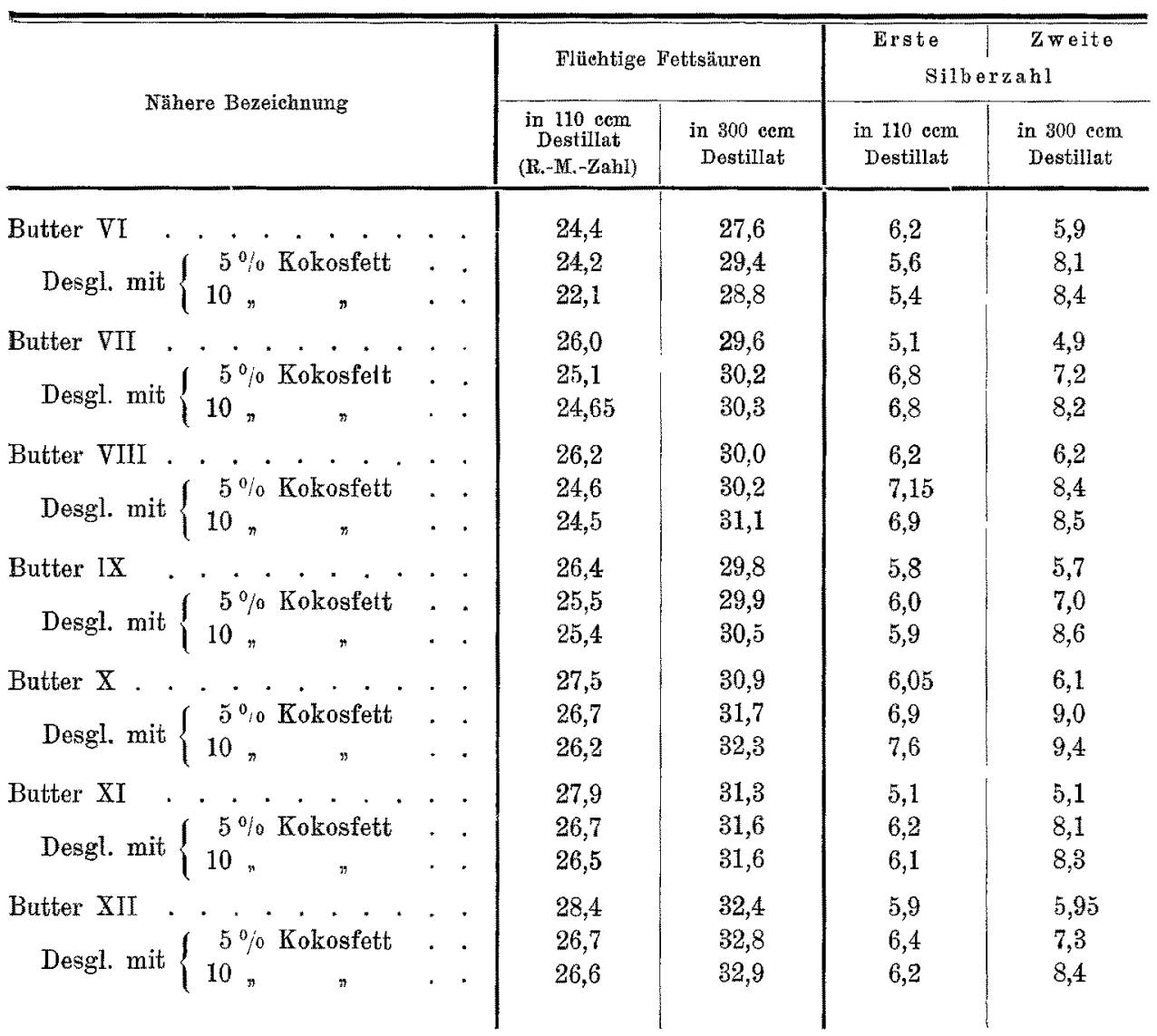

Aus diesen Untersuchungsergebnissen ist ersichtlich, daß bei sämtlichen Proben von reinem Butterfett die zweite Silberzahl gleich oder niedriger ist als die erste; in zwei Fällen wurde eine Steigerung von 0,05 gefunden, diese fällt aber innerhalb der Fehlergrenzen. Dagegen ergab schon eine Beimischung von nur $5 \%$ Kokosfett eine sehr deutliche Erhöhung der zweiten Silberzahl. Wir können also Jensen völlig beipflichten, wenn er behauptet, daß schon diese Menge an Kokosfett durch den Gehalt an Kaprylsäure qualitativ mit Sicherheit nachzuweisen sei. Nur hoffen wir durch unsere Arbeit dem Verfahren eine praktische Form gegeben zu haben, welche sich den Bedürfnissen und den sonst üblichen Methoden der Butteruntersuchung in leicht faßbarer und sachgemäßer Weise anschließt.

Wir wissen aber nur zu gut, daß allein die Praxis auf die Dauer über die Verwendbarkeit einer Butterprüfungsmethode ein Urteil abzugeben vermag und möchten daher nicht verfehlen, die Fachgenossen aufs dringlichste zu bitten, die Methode nachzuprüfen und ibre Erfahrungen bekannt zu geben. 